\title{
Procedural Implementation and Axiomatization of the Weighted Nonseparable Cost Value
}

\author{
Hui Yang $\mathbb{D},{ }^{1,2}$ Wenna Wang, ${ }^{3}$ and Zhengsheng Ding ${ }^{2}$ \\ ${ }^{1}$ School of Natural and Applied Sciences, Northwestern Polytechnical University, Xi'an 710072, China \\ ${ }^{2}$ School of Science, Xi'an University of Science and Technology, Xi'an 710054, China \\ ${ }^{3}$ Department of Financial Mathematics, Xi'an University of Finance and Economics, Xi'an 710100, China \\ Correspondence should be addressed to Hui Yang; yangh816@mail.nwpu.edu.cn
}

Received 11 October 2019; Accepted 23 April 2020; Published 7 June 2020

Academic Editor: Massimiliano Ferrara

Copyright $\odot 2020$ Hui Yang et al. This is an open access article distributed under the Creative Commons Attribution License, which permits unrestricted use, distribution, and reproduction in any medium, provided the original work is properly cited.

\begin{abstract}
The paper defines a new value called the weighted nonseparable cost value (weighted-NSC value), which divides the nonseparable cost on the ground of an exogenous attached weight and compromises egalitarianism and utilitarianism of a value flexibly. First, we construct an optimization model to minimize the deweighted variance of complaint and define its optimal solution to be the weighted-NSC value. Second, a process is set up to acquire the weighted-NSC value, which enlarges the traditional procedural values. In the process, one player's marginal contribution is divided up by all participants rather than merely restricted within his precursors. Lastly, adopting the weight in defining a value destructs the classical symmetry. This promotes the definition of $\omega$-symmetry for the grand-marginal normalized game to defend against the effect of weight and axiomatically sculptures the weighted-NSC value. Dual dummifying player property is also applied to characterize the new defined value.
\end{abstract}

\section{Introduction}

Consider a wireless network with $n$ nodes. The nodes communicate with far-off destinations through the cooperation of the intermediate nodes. Many indexes of the network, such as throughput and lifetime, will be raised dramatically, and the communication cost be reduced greatly through cooperation [1]. The corresponding payoff allocation or the cost distribution problem is of highly interest $[2,3]$. Cooperative game theory offers a variety of values to address how to fairly distribute the overall payoff in many fields such as economics [4] and networks [5].

The eminent Shapley value [6] allocates to each participant his expected payoff on all possible orders. In the permutations, each newcomer possesses the whole marginal contribution, which is utilitarian. Averaging the total worth is a natural way for allocation problems, which is entirely egalitarian. The corresponding value is thereby named as the equal division value (ED value) in cooperative games.
The egalitarian nonseparable cost value (ENSC value, [7]) at first gives everyone his grand marginal contribution and afterwards averages the (positive or negative) nonseparable part to everyone. Sun et al. [8] revealed that the ENSC value embodies utilitarianism in virtue of the player's attitude towards the grand marginal contributions. Then, we encounter the issue of equilibrate utilitarianism and egalitarianism during allocation process.

Ruiz et al. [9] initially linked the optimization theory with allocation issues in cooperative games. They innovatively harnessed the least square value to minimize the variance of all players' complaint. Triggered by Ruiz, Wang et al. [10] proposed the ideal values, targets at narrowing the gap between one's given payoff and expected rewards. This paper constructs an optimization model considering the deweighted variance of complaint. Through deriving the unique optimal solution, we define the weighted nonseparable cost value (weighted-NSC value). 
Endowing a value with weight is a common path to reconcile the relationship between egalitarianism and utilitarianism. Aristotle's maxim, "Equals should be treated equally, and unequals unequally, in proportion to relevant similarities and differences" stresses the fair allocation should consider players' similarities and differences, which could be reflected by the weight. In addition, the weight coefficient sheds light on participants' importance, power, or negotiation ability. The weighted Shapley value is defined in [11] and be portrayed axiomatically by Kalai and Samet [12]. Yang et al. [13] researched the weighted surplus division value in cooperative games. In this paper, the weighted-NSC value is defined applying the exogenous determined positive weight. Based on the definition, the nonseparable part is assigned to players according to their importance, which can be evaluated by different methods and be adjusted flexibly.

Procedural value [14] is another efficient method to compromise utilitarianism and egalitarianism on an allocation rule in the reason that one's marginal contribution in a given permutation will be shared with his precursor. Sun et al. [8] provided a process to achieve the ENSC value. This article designs an enlarged process to present a more detailed research of the weighted-NSC value. The philosophy idea of our process differs from the process in [14] because the marginal contributions are shared by all players instead of just among the precursor.

Axiomatization presents an effective method to sculpture a value. The weighted-NSC value links each player's payoff with a weight coefficient and logically does not conform to the axiom of symmetry anymore. This evokes us to propose $\omega$-symmetry for the grand-marginal normalized game in order to characterize the weighted-NSC value. The covariance can also depict the weighted-NSC value from a mapping perspective. Ultimately, the dual dummifying player property is proposed to characterize the weightedNSC value.

We structure the article as follows. Section 2 briefly lists the fundamental notions of cooperative games. Section 3 puts forward the weighted-NSC value and presents an optimization explanation for it through tackling an optimization model. The procedural implementation for this value is provided in Section 4 by means of designing a process. Section 5 engages in axiomatic description of the weightedNSC value applying several new proposed properties. Section 6 is committed to a general summary of the article and sketching of the potential aspect of further research.

\section{Preliminaries}

A player set $N$ and a characteristic function $v: 2^{N} \longrightarrow R$ altogether are contributing factors to characterize a cooperative game, written as $\langle N, v\rangle$. Without confusion, we abbreviate it as a game and correspondingly denote as $v$. Here, $2^{N}$ denotes the power set of $N$. Given any coalition $S \in 2^{N} \backslash\{\phi\}$, the characteristic function designates $S$ the worth $v(S)$, trivially with $v(\phi)=0$. The cardinal of the coalition $S$ is labelled by $s$. The set of all games on $N$ is written as $G^{N}$.
For $v_{1}, v_{2} \in G^{N}$ and real number $p \in R$, the games $v_{1}+v_{2}$ and $p \cdot v_{1}$ are, respectively, given by $\left(v_{1}+v_{2}\right)(S)=v_{1}(S)+$ $v_{2}(S)$ and $\left(p \cdot v_{1}\right)(S)=p \cdot v_{1}(S)$, for $S \subseteq N$. A game $v \in G^{N}$ is named weakly essential when $\sum_{k \in N} v(\{k\}) \leq v(N)$ and be called inessential when $v(S)=\sum_{k \in S} v(\{k\})$ holds for $S \subseteq N$. Given $k, j \in N$, if $v(S \cup\{k\})=v(S \cup\{j\})$, for $S \subseteq N \backslash\{k, j\}$, we call them symmetric players.

We view a payoff vector (or payoff) as an $n$-dimensional vector $x=\left(x_{k}\right)_{k \in N} \in R^{n}$ and incidentally define $x(S)=\sum_{k \in S} x_{k}$ for $S \subseteq N$. If a payoff $x$ possesses $x(N)=v(N)$, we name it efficient and denote the preimputation set by $I^{*}(v)=\left\{x \in R^{n} \mid x(N)=v(N)\right\}$, for $v \in G^{N}$. Formally, a mapping $\phi: G^{N} \longrightarrow R^{n}$ determines a value which affords each $v \in G^{N}$ a payoff, $\phi(v)=\left(\phi_{k}(v)\right)_{k \in N} \in R^{n}$. The component $\phi_{k}(v)$ invariably can be regarded as an evaluation of player $k$ 's benefit when taking part in the game $v$.

The well-known Shapley value [6] distributes to each participant the expected marginal contribution, which is defined by the formula as follows:

$$
\mathrm{Sh}_{k}(v)=\sum_{S \subseteq N, S \ni k} \frac{(n-s) !(s-1) !}{n !}[v(S)-v(S \backslash\{k\})], \quad \text { for } k \in N .
$$

The mean value of $v(N)$ provides the concept of the equal division value (ED value):

$$
\mathrm{ED}_{k}(v)=\frac{1}{n} v(N), \quad \text { for } k \in N
$$

The egalitarian nonseparable cost value (ENSC value [7]) allocates the nonseparable part equally after giving each one his grand marginal contribution. Given any $v \in G^{N}$,

$$
\mathrm{ENSC}_{k}(v)=b_{k}^{v}+\frac{1}{n}\left[v(N)-\sum_{j \in N} b_{j}^{v}\right], \quad \text { for } k \in N
$$

where $b_{k}^{v}=v(N)-v(N \backslash\{k\})$ is player $k$ 's grand marginal contribution.

Take a coefficient $0 \leq \alpha \leq 1$, and the $\alpha$-ENSC value [15] is portrayed by convex combination, $\alpha \operatorname{ENSC}(v)+(1-\alpha)$ $\operatorname{ED}(v)$, which is equivalent to the following formula:

$$
\operatorname{ENSC}_{k}^{\alpha}(v)=\alpha b_{k}^{v}+\frac{1}{n}\left[v(N)-\sum_{j \in N} \alpha b_{j}^{v}\right], \quad \text { for } k \in N .
$$

Let $\phi: G^{N} \longrightarrow R^{n}$ be a value, and we enumerate the wellknown axioms:

(i) Efficiency $(E): \sum_{k \in N} \phi_{k}(v)=v(N)$, for all $v \in G^{N}$

(ii) Symmetry $(S): \phi_{k}(v)=\phi_{j}(v)$, for symmetric players $k, j \in N$ in $v \in G^{N}$

(iii) Individual rationality (IR): $\phi_{k}(v) \geq v(\{k\})$, for all weakly essential game $v \in G^{N}$

(iv) Additivity $(A): \phi_{k}\left(v_{1}+v_{2}\right)=\phi_{k}\left(v_{1}\right)+\phi_{k}\left(v_{2}\right)$, for all $v_{1}, v_{2} \in G^{N}$

(v) Linearity (L): $\phi_{k}\left(c v_{1}+d v_{2}\right)=c \phi_{k}\left(v_{1}\right)+d \phi_{k}\left(v_{2}\right)$, for all $v_{1}, v_{2} \in G^{N}$ and $c, d \in R$ 
(vi) Inessential game property (IN): $\phi_{k}(v)=v(\{k\})$, for all inessential game $v \in G^{N}$

\section{The Weighted Nonseparable Cost Value and Its Optimization Implementation}

The grand marginal contribution of player $i, b_{i}^{v}$, is testified to be the supremum of the core [16]. Consequently, all players in a game will aspire to $b_{i}^{v}$ and prefer the allocation which is close to $b_{i}^{v}$. Given $x \in I^{*}(v)$, player $i$ 's complaint $e(i, x)=b_{i}^{v}-x_{i}$ is adopted as a rational measure of $i$ 's dissatisfaction.

Invariably, we could not decrease everyone's complaint simultaneously. According to the egalitarian philosophy, we strive to find out an allocation to balance each one's complaint instead of merely push down the highest complaint. The variance of the complaint is applicable to evaluate fluctuation degree of players' complaint.

Furthermore, we might confront the situation that every player has different importance and be treated differently. This evokes us that the weight coefficient needs to be taken into account during inspecting the variance of the complaint. Given the weight system $\Delta^{n}:=\left\{\omega \in R_{++}^{n}\right.$ $\left.\mid \sum_{i \in N} \omega_{i}=1\right\}$, we modify the traditional variance and define the deweighted variance as $\sum_{i \in N}\left(1 / \omega_{i}\right)\left(b_{i}^{v}-x_{i}\right)^{2}$ to equilibrate the complaint of each player ("deweighted" here clarifies removing the effect of the weight). The detailed study of the deweighted variance is unfolded via an optimization model as follows. Given any game $v \in G^{N}$ and weight vector $\omega \in \Delta^{n}$,

$$
\begin{aligned}
& \text { model I : } \min _{x \in R^{n}} \sum_{i \in N} \frac{1}{\omega_{i}}\left(b_{i}^{v}-x_{i}\right)^{2} \\
& \text { s.t. } \quad \sum_{i \in N} x_{i}=v(N) .
\end{aligned}
$$

Explicitly, equation (5) presents a convex optimization problem. Its optimal solution is either unattainable or exists uniquely. To seek model I's optimum solution, we formulate its Lagrange function as follows:

$$
L(x, \lambda)=\sum_{i \in N} \frac{1}{\omega_{i}}\left(b_{i}^{v}-x_{i}\right)^{2}+\lambda\left[\sum_{i \in N} x_{i}-v(N)\right] .
$$

Then, calculate the partial derivative of $L(x, \lambda)$ with $x_{i}$, $i \in N$, and we generate Lagrange conditions:

$$
L_{x_{i}}(x, \lambda)=\frac{2}{\omega_{i}}\left(x_{i}-b_{i}^{v}\right)+\lambda=0 .
$$

Furthermore, we demonstrate the constraint of efficiency through computing the partial derivative of equation (6) with $\lambda$ :

$$
L_{\lambda}(x, \lambda)=\sum_{i \in N} x_{i}-v(N)=0 .
$$

Combining equations (7) and (8), model I's unique optimal solution is

$$
x_{i}^{*}=b_{i}^{v}+\omega_{i}\left[v(N)-\sum_{j \in N} b_{j}^{v}\right], \quad \text { for } i \in N .
$$

Consequently, a new value of allocating the payoff emerges in our mind.

Definition 1. For any game $v \in G^{N}$ and weight vector $\omega \in \Delta^{n}$, the weighted nonseparable cost value (weightedNSC value) is defined as

$$
\mathrm{NSC}_{i}^{\omega}(v)=b_{i}^{v}+\omega_{i}\left[v(N)-\sum_{j \in N} b_{j}^{v}\right], \quad \text { for } i \in N,
$$

where $b_{i}^{v}$ represents player $i$ 's grand marginal contribution.

We name it the weighted nonseparable cost value because $b_{i}^{v}$ and $v(N)-\sum_{j \in N} b_{j}^{v}$ are, respectively, called the separable cost and nonseparable cost [7]. After giving player $i$ his separable cost, the weighed-NSC value divides the remaining nonseparable part among all players based on a weight vector $\omega \in \Delta^{n}$, while the ENSC value distributes this part equally to all players. The weight reflects a player's bargaining ability or accounts for a player's probability of forming coalitions with others. In practical application, the weight can be altered flexibly using different methods. Model I offers an implementation process for the weighted-NSC value. Without more ado, we get the following conclusion based on the derivation process of model I.

Theorem 1. Given any game $v \in G^{N}$ and weight vector $\omega \in \Delta^{n}$, model I's optimal solution exists uniquely and equals to the weighted-NSC value:

$$
x_{i}^{*}=b_{i}^{v}+\omega_{i}\left[v(N)-\sum_{j \in N} b_{j}^{v}\right]=\mathrm{NSC}_{i}^{\omega}(v), \quad \text { for } i \in N .
$$

Setting aside the weight coefficient, we derive the following corollaries. Given any $v \in G^{N}$,

$$
\begin{aligned}
& \text { model II : } \min _{x \in R^{n}} \sum_{i \in N}\left(b_{i}^{v}-x_{i}\right)^{2} \\
& \text { s.t. } \quad \sum_{i \in N} x_{i}=v(N) .
\end{aligned}
$$

Corollary 1. Given any game $v \in G^{N}$, model II's optimal solution exists uniquely and equals to the ENSC value:

$$
x_{i}^{*}=b_{i}^{v}+\frac{1}{n}\left[v(N)-\sum_{j \in N} b_{j}^{v}\right] \text {, for } i \in N .
$$

Identically, take $v \in G^{N}$ and $0 \leq \alpha \leq 1$ :

$$
\begin{aligned}
& \text { model III : } \min _{x \in R^{n}} \sum_{i \in N}\left(\alpha b_{i}^{v}-x_{i}\right)^{2} \\
& \text { s.t. } \sum_{i \in N} x_{i}=v(N) .
\end{aligned}
$$


Corollary 2. Given any game $v \in G^{N}$, model III's optimal solution exists uniquely and equals to the $\alpha$-ENSC value, i.e.,

$$
x_{i}^{*}=\alpha b_{i}^{v}+\frac{1}{n}\left[v(N)-\sum_{j \in N} \alpha b_{j}^{v}\right], \quad \text { for } i \in N \text {. }
$$

Take $v \in G^{N}, \omega \in \Delta^{n}$, and $x \in I^{*}(v)$, and we link each $e(i$, $x$ ) with $\omega_{i}$ and put forward player $i$ 's deweighted complaint as

$$
e^{\omega}(i, x)=\frac{1}{\omega_{i}}\left(b_{i}^{v}-x_{i}\right)
$$

Let $\phi: G^{N} \longrightarrow R^{n}$; we propose the corresponding axiom as follows:

(i) Equal deweighted complaint property (EC): for all $v \in G^{N}$ and $\omega \in \Delta^{n}$, the value $\phi$ verifies that

$$
e^{\omega}(i, \phi)=e^{\omega}(j, \phi), \quad \text { for } i, j \in N \text {. }
$$

Based on equation (10), it is easy to assert the following for any $v \in G^{N}$ and $\omega \in \Delta^{n}$.

Theorem 2. A value $\phi: G^{N} \longrightarrow R^{n}$ possesses (E) and (EC) if and only if $\phi(v)=N S C^{\omega}(v)$.

\section{The Procedural Explanation}

Procedural values [14] are hinged on a process which settles how to divide a player's marginal contribution among his predecessors in a given permutation. When a participant intends to collaborate with existing players, a fraction of his marginal contribution is compelled to be handed over and be shared with these people. Motivated by the philosophy of egalitarianism, this paper modifies the conventional process creatively and designs an extended process to obtain the weighted-NSC value.

For any $v \in G^{N}$ is denoted $T(N)$ as all possible orders of $N$. Given an order $\tau \in T(N), \tau(k)$ is the player who is at position $k$ in this order. Player $i$ 's precursor (including $i$ ) under $\tau$ is defined as follows:

$$
p_{i}^{\tau}:=\left\{\tau(k) \in N \mid k \leq \tau^{-1}(i)\right\}
$$

\subsection{Process 1}

Step 1: the participants set up permutations at random, and each $\tau$ in $T(N)$ is equal possible

Step 2: for player $i(1 \leq i \leq n)$, his precursor set is established as referred in equation (18), and the value $v\left(p_{i}^{\tau}\right)-v\left(p_{i}^{\tau} \backslash\{i\}\right)$ is produced as the marginal contribution

Step 3: player $i$ claims his grand marginal contribution $b_{i}^{v}$

Step 4: the surplus (or deficit) after player $i$ claim in step 3, $v\left(p_{i}^{\tau}\right)-v\left(p_{i}^{\tau} \backslash\{i\}\right)$ is divided up by each $i \in N$ based on the weight $\omega \in \Delta^{n}$
Step 5: compute $i$ expected payoff on all orders in $T(N)$

In step 4 of Process 1 , the surplus (or deficit) is placed into a pool after $b_{i}^{v}$ is taken away. In contrast to [14], our process not only broadens the allocation object from $p_{i}^{\tau}$ to $N$ but also links the weight with benefit allocation. The philosophy idea in Process 1 is analogous to the redistribution of social welfare, i.e., after each person gets his or her own salary, the excess part will be taxed according to the given rate by Tax Bureau. In this sense, this process offers a significant tool for social welfare allocation. We illustrate Process 1 by an example.

Example 1. Given players' weight vector $\omega=\left\{\omega_{1}, \omega_{2}, \omega_{3}\right\}$ and the entrance order $\tau=(2,3,1) \in T(N)$.

(1) Firstly, player 2 joins the game. He charges his grand marginal contribution $b_{2}^{v}$, and then, all players, $i \in\{1,2,3\}$, divide up the nonseparable part $v(\{2\})-$ $b_{2}^{v}$ on account of the weight $\omega$.

(2) Then, there comes player 3 right after player $2, \mathrm{He}$ also claims his own grand marginal contribution $b_{3}^{v}$, and the surplus is distributed by all players rather than just his predecessor based on the weight $\omega$.

(3) Lastly, player 1 enters the coalition already present. Similarly, the nonseparable cost is allocated with the weight $\omega$ after player 1 asks for $\tau=(2,3,1)$.

Table 1 demonstrates the allocation result in every step and the total payoff to players under the permutation $\tau=(2,3,1)$.

Obviously, each one's payoff is irrelevant to permutations; therefore, the expected payoff in all orders of the arrival is the same as in Table 1, which is

$$
\phi_{i}(v)=b_{i}^{v}+\omega_{i}\left[v(N)-\sum_{j \in N} b_{j}^{v}\right], \quad \text { for } i=1,2,3 .
$$

From this example, we automatically deduce the following conclusion and attach a detailed precise proof for it.

Theorem 3. Given any game $v \in G^{N}$ and weight system $\omega \in \Delta^{n}$, the expected payoff $\phi^{\omega}(v)$ calculated by Process 1 equals to the weighted-NSC value.

Proof. For any $v \in G^{N}$ and $\omega \in \Delta^{n}$, randomly take an order $\tau \in T(N)$, and player $i$ payoff $\phi_{i, \tau}^{\omega}(v)$ in the above process is determined as

$$
\phi_{i, \tau}^{\omega}(v)=b_{i}^{v}+\omega_{i} \sum_{k=1}^{n}\left[v\left(p_{\tau(k)}^{\tau}\right)-v\left(p_{\tau(k)}^{\tau} \backslash \tau(k)\right)-b_{\tau(k)}^{v}\right] .
$$

Therefore, the expect value $\phi^{\omega}(v)$ in step 5 is

$$
\phi_{i}^{\omega}(v)=\frac{1}{n !} \sum_{\tau \in T(N)} \phi_{i, \tau}^{\omega}(v), \quad \text { for } i \in N .
$$

Based on equality (20), the following formula can be derived: 
TABle 1: Payoff for players under procedure $\tau=(2,3,1)$.

\begin{tabular}{lccr}
\hline$N$ & 2's payoff in every step & 3's payoff in every step & 1's payoff in every step \\
\hline 2 joins in & $b_{2}^{v}+\omega_{2}\left[v(\{2\})-b_{2}^{v}\right]$ & $\omega_{3}\left[v(\{2\})-b_{2}^{v}\right]$ & $\omega_{1}\left[v(\{2\})-b_{2}^{v}\right]$ \\
3 joins in & $\omega_{2}\left[v(\{2,3\})-v(\{2\})-b_{3}^{v}\right]$ & $b_{3}^{v}+\omega_{3}\left[v(\{2,3\})-v(\{2\})-b_{3}^{v}\right]$ & $\omega_{1}\left[v(\{2,3\})-v(\{2\})-b_{3}^{v}\right]$ \\
1 joins in & $\omega_{2}\left[v(N)-v(\{2,3\})-b_{1}^{v}\right]$ & $\omega_{3}\left[v(N)-v(\{2,3\})-b_{1}^{v}\right]$ & $b_{i}^{v}+\omega_{1}\left[v(N)-v(\{2,3\})-b_{1}^{v}\right]$ \\
Overall payoff & $b_{2}^{v}+\omega_{2}\left[v(N)-\sum_{j \in N} b_{j}^{v}\right]$ & $b_{3}^{v}+\omega_{3}\left[v(N)-\sum_{j \in N} b_{j}^{v}\right]$ & $b_{1}^{v}+\omega_{1}\left[v(N)-\sum_{j \in N} b_{j}^{v}\right]$ \\
\hline
\end{tabular}

$$
\phi_{i, \tau}^{\omega}(v)=b_{i}^{v}+\omega_{i}\left[v(N)-\sum_{k=1}^{n} b_{\tau(k)}^{v}\right] .
$$

In essence, the right side of equality (20) is independent of the permutation; hence,

$$
\phi_{i}^{\omega}(v)=b_{i}^{v}+\omega_{i}\left[v(N)-\sum_{j \in N} b_{j}^{v}\right]=\mathrm{NSC}_{\mathrm{i}}^{\omega}(v) .
$$

This theorem offers a procedural explanation for the weighted-NSC value and incidentally gives rise to the following remarks evidently.

Remark 1. Given any $v \in G^{N}$, if step 4 in Process 1 is executed presuming that players are of equal importance, i.e., $\omega_{i}=1 / n$, the expected value in step 5 is exactly the ENSC value.

Remark 2. Given any $v \in G^{N}$, if in step 3 of Process 1 , player $i \in N$ claims $\alpha b_{i}^{v}(\alpha \in[0,1])$ and step 4 is executed presuming that players are of equal importance, i.e., $\omega_{i}=1 / n$, the expected value in step 5 is exactly the $\alpha$-ENSC value.

\section{Axiomatizations}

Axiomatization is a fundamental method to depict a solution in cooperative games. The axiom of symmetry is applied to describe the ENSC value in [15]. The weight coefficient in the definition of $\operatorname{NSC}^{\omega}(v)$ contributes to asymmetry. If players still pursue equitable treatment after deleting the influence of weight, it is necessary to generate a new symmetry property which is restricted in a special kind of game.

Definition 2. A game $v \in G^{N}$ is defined as a grand-marginal normalized game if $b_{i}^{v}=0$ for all $i \in N$.

(i) $\omega$-symmetry for grand-marginal normalized game ( $\omega$-SYM): the value $\phi: G^{N} \longrightarrow R^{n}$ verifies that

$$
\frac{\phi_{i}(v)}{\omega_{i}}=\frac{\phi_{j}(v)}{\omega_{j}}, \quad i, j \in N
$$

where $v \in G^{N}$ is a grand-marginal normalized game and $\omega \in \Delta^{n}$

This axiom indicates that players will be awarded equally in the grand-marginal normalized games if we do not consider the factor of weight. Apparently, the weighted-NSC value does not abide by the classical symmetry anymore while complies with $\omega$-SYM, and we dedicate $\omega$-SYM to describe the weighted-NSC value. Given any $v \in G^{N}$ and $\omega \in \Delta^{n}$, the following can be obtained.

Theorem 4. A value $\phi: G^{N} \longrightarrow R^{n}$ possesses (E), (A), (IN), and $(\omega-S Y M)$ if and only if $\phi(v)=N S C^{\omega}(v)$.

Proof

(1) Take $\omega \in \Delta^{n}$; it is trivial to testify that the weightedNSC value abides by $(E),(A),($ IN), and $(\omega$-SYM).

(2) Proof of uniqueness: let $\phi: G^{N} \longrightarrow R^{n}$ be a value with $(E),(A),(\mathrm{IN})$, and ( $\omega$-SYM). Given $v \in G^{N}$, we construct

$$
v^{0}(S):=v(S)-\sum_{j \in S} b_{j}^{v}, \quad \text { for } S \subseteq N .
$$

Then, $v^{0}$ is a grand-marginal normalized game because $b_{i}^{v^{0}}=v^{0}(N)-v^{0}(N \backslash\{i\})$ holds. Applying the property $(\omega$-SYM), the following equation is obtained:

$$
\omega_{i} \phi_{j}\left(v^{0}\right)=\omega_{j} \phi_{i}\left(v^{0}\right), \quad \text { for } \mathrm{i}, \mathrm{j} \in \mathrm{N} .
$$

Summing up all $j \in N$ in equation (26), we can get

$$
\phi_{i}\left(v^{0}\right)=\omega_{i} \sum_{j \in N} \phi_{j}\left(v^{0}\right) .
$$

Combined with the efficiency, we have

$$
\phi_{i}\left(v^{0}\right)=\omega_{i} v^{0}(N)=\omega_{i}\left[v(N)-\sum_{j \in N} b_{j}^{v}\right] .
$$

Let $\widetilde{v}$ : $=v-v^{0}$, which is obviously an inessential game. Owing to the property (IN), it is easy to derive $\phi_{i}(\widetilde{v})=\widetilde{v}(\{i\})-b_{i}^{v}$. Because $v=\widetilde{v}+v^{0}$ is involved with additivity,

$$
\phi_{i}(v)=\phi_{i}(\widetilde{v})+\phi_{i}\left(v^{0}\right)=\operatorname{NSC}_{\mathrm{i}}^{\omega}(v) .
$$

Remark 3. In Theorem 4, the axiom $(L)$ is enough to substitute for $(A)$ according to the above proof.

Different from the axioms of $(A)$ and $(L)$, covariance researches a value through the angle of mapping:

(ii) Covariance (COV): $\phi_{i}(c v+d)=c \phi_{i}(v)-d_{i}$, for all $v \in G^{N}, \quad c \in R, \quad$ and $\quad d \in R^{n}$, where $(c v+d)(S)=c v(S)+\sum_{j \in S} d_{j}$, for $S \subseteq N$.

Alternatively, we can apply this property to depict the weighted-NSC value. 
Corollary 3. A value $\phi: G^{N} \longrightarrow R^{n}$ possesses (E), (L), $(C O V)$, and ( $\omega-S Y M)$ if and only if $\phi(v)=N S C^{\omega}(v)$.

Proof

(1) Given any $\omega \in \Delta^{n}$ obviously, the weighted-NSC value verifies $(E),(L),(\mathrm{COV})$, and ( $\omega$-SYM).

(2) Proof of uniqueness: let $\phi: G^{N} \longrightarrow R^{n}$ be a value with $(E),(L),(\mathrm{COV})$, and ( $\omega$-SYM). We concentrate on the inessential game $u \in G^{N}, u(S)=\sum_{j \in S} u(\{j\})$. Using (COV), $\phi_{i}(u)=u(\{i\})$. This states that also $\phi$ possesses the axiom (IN) and then $\phi$ coincides with the weighted-NSC value combined with Theorem 4 .

One is dummifying when the coalition (including this player) worth equals to the cumulative of each member's individual worth. Dummifying player's presence in a coalition hinders cooperation to some extent. Giving a dummifying player his individual worth same to be a plausible distribution. The player who brings dummifying influence to coalitions will be researched in a dual way.

Definition 3. For any game $v \in G^{N}$, player $i$ is named as a dual dummifying player if $v(N)-(N \backslash S)=\sum_{k \in S} b_{k}^{v}$, for $S \subseteq N$ and $S \in i$.

Relevant axiom is put forward as follows:

(iii) Dual dummifying player property (DD): $\phi_{i}(v)=b_{i}^{v}$, for all $v \in G^{N}$, and dual dummifying player $i \in N$.

Generally, the grand marginal contributions cannot be guaranteed. However, this property insures this ideal payoff for the dual dummifying player. We depict the weightedNSC value with the dual dummifying player property as follows. Given any $v \in G^{N}$ and $\omega \in \Delta^{n}$, the following can be obtained.

Theorem 5. A value $\phi: G^{N} \longrightarrow R^{n}$ possesses (E), (L), (DD), and $(\omega-S Y M)$ if and only if $\phi(v)=N S C^{\omega}(v)$.

\section{Proof}

(1) Given any $\omega \in \Delta^{n}$, the weighted-NSC value explicitly satisfies $(E),(L)$, and ( $\omega$-SYM), and we now check (DD).

Given any $v \in G^{N}$, take a dual dummifying player $i \in N$ randomly, and we attain $v(N)=\sum_{j \in N} b_{j}^{v}$ owing to Definition 3. Consequently, we compute $\operatorname{NSC}_{i}^{\omega}(v)=b_{i}^{v}, i \in N$. This exactly verifies DD.

(2) Proof of uniqueness: let $\phi: G^{N} \longrightarrow R^{n}$ be a value with $(E),(L),(\mathrm{DD})$, and ( $\omega$-SYM). For any $v \in G^{N}$, $c \in R$, and $d \in R^{n}$ and according to the property $(L)$ of $\phi$,

$$
\phi_{i}(c v+d)=c \phi_{i}(v)+\phi_{i}(d), \quad \text { for } i \in N,
$$

where $d(S)=\sum_{i \in S} d_{i}$, for $S \subseteq N$. Obviously,

$$
b_{i}^{d}=d(N)-d(N \backslash\{i\})=d_{i} .
$$

Then,

$$
d(N)-d(N \backslash S)=\sum_{k \in S} d_{k}=\sum_{k \in S} b_{k}^{d}, \quad \text { for } S \subseteq N .
$$

Therefore, each $i \in N$ is a dual dummifying player in $d$. Since $\phi_{i}(d)=d_{i}$ holds due to (DD), we deduce that

$$
\phi_{i}(c v+d)=c \phi_{i}(v)+d_{i}, \quad \text { for } i \in \mathrm{N},
$$

which shows that $\phi$ satisfies (COV). With Corollary 3, we can easily verify uniqueness.

Example 2. Assume a partial wireless network is composed of 3 nodes as in Figure 1. Node $n_{1}$ contributes nothing to any coalition, and node $n_{1}$ and node $n_{3}$ communicate through node $n_{2}$. If a node is located within the communication range of another one, there is a link between them.

The situation could be formulated in terms of a cooperative game with characteristic function. Presume that each node's value is as follows:

$$
\begin{aligned}
& v(\phi)=0, \\
& v\left(\left\{n_{1}\right\}\right)=1, \\
& v\left(\left\{n_{2}\right\}\right)=2, \\
& v\left(\left\{n_{3}\right\}\right)=3, \\
& v\left(\left\{n_{1}, n_{2}\right\}\right)=3, \\
& v\left(\left\{n_{1}, n_{3}\right\}\right)=4, \\
& v\left(\left\{n_{2}, n_{3}\right\}\right)=8, \\
& v\left(\left\{n_{1}, n_{2}, n_{3}\right\}\right)=9 .
\end{aligned}
$$

Table 2 lists the calculation results of the payoff distribution according to different values.

(1) Node $n_{1}$, as a dummy player, only gets its individual value by the Shapley value.

(2) The ED value distributes the total payoff evenly among all the players regardless of the contribution difference of each node to the wireless networks, which embodies total egalitarianism.

(3) The weighted-NSC value equals to the ENSC value if the weight $\omega_{i}=1 / n$. Note that the dummy player gets nothing in the game which verifies that the ENSC value is more utilitarian than the Shapley value. In the second condition, the weight, $\omega_{i}=b_{i}^{v} / \sum_{j \in N} b_{j}^{v}$, is proportional to the grand marginal contribution. The third weight $\omega_{i}$ is proportional to each one's average marginal contribution to any coalition. Note that the payoff $\{3 / 4,15 / 4,18 / 4\}$ is the closest to Shapley value than others.

Evidently, the weighted-NSC value provides a rational payoff distribution rule which can flexibly balance the relationship between utilitarianism and egalitarianism during allocation process according to actual situations. 


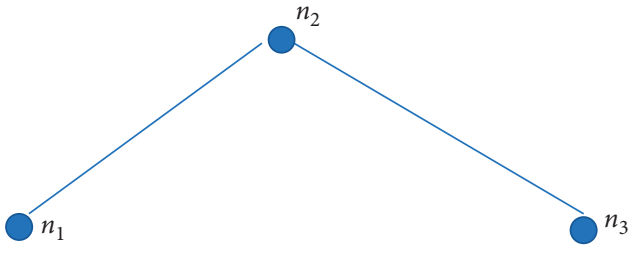

FIGURE 1: A wireless network with three nodes.

TABLE 2: The payoff distributions of 3 nodes.

\begin{tabular}{ccccc}
\hline & & Node $n_{1}$ & Node $n_{2}$ & Node $n_{3}$ \\
\hline & $\operatorname{Sh}(v)$ & 1 & $7 / 2$ & $9 / 2$ \\
$\operatorname{NSC}^{\omega}(v)$ & $\omega=\{1 / 3,1 / 3,1 / 3\}$ & 3 & 3 & 3 \\
& $\omega=\{1 / 12,5 / 12,6 / 12\}$ & $3 / 4$ & $15 / 4$ & $18 / 4$ \\
& $\omega=\{2 / 18,7 / 18,9 / 18\}$ & $4 / 6$ & $23 / 6$ & $27 / 6$ \\
\hline
\end{tabular}

\section{Conclusions}

The weighted-NSC value defined in this article compromises the principle of equalitarianism and utilitarianism with given exogenous weight vector in the allocation of payoff. In contrast to the optimization model of the ENSC value and the $\alpha$-ENSC value, we define the deweighted variance of complaint and set up the corresponding model to attain the weighted-NSC value. Furthermore, we devise a detailed process to obtain the weighted-NSC value, which is similar but different to the traditional procedural values in terms of allocation objects of the marginal contribution. In our process, players' marginal contribution is divided by all the participants of the game. In addition, we portray the weighted-NSC value axiomatically applying new defined axioms.

Inspired by the geometrical study of cooperative games in [17], the weighted-NSC value can be introduced into graph-restricted games and help to research the topological structure of wireless networks, which will probably improve the connectivity performance of wireless networks. In addition, applying the weighted value in cooperative games to the sustainable supply chain management [18] is worthy of further research.

\section{Data Availability}

All data used to support the findings of this study are included within the article.

\section{Conflicts of Interest}

The authors declare no conflicts of interest.

\section{Acknowledgments}

The authors are grateful to Xueli Song for many helpful discussions. This work was partially funded by Shaanxi Science and Technology Research and Development
Program (no. 2018JM1047) and the National Natural Science Foundation of China (nos. 71571143 and 11601417).

\section{References}

[1] R. Casado-Vara, F. Prieto-Castrillo, and J. M. Corchado, "A game theory approach for cooperative control to improve data quality and false data detection in WSN," International Journal of Robust and Nonlinear Control, vol. 28, no. 16, pp. 5087-5102, 2018.

[2] C. Li and H. Xue, "Model of profit allocation based on generalized solution in network cooperative game," Control and Decision, vol. 32, no. 6, pp. 1041-1046, 2017.

[3] C. Singh, S. Sarkar, A. Aram, and A. Kumar, "Cooperative profit sharing in coalition-based resource allocation in wireless networks," IEEE/ACM Transactions on Networking, vol. 20, no. 1, pp. 69-83, 2012.

[4] P. Beraldi, A. Violi, M. Ferrara, C. Ciancio, and B. A. Pansera, "Dealing with complex transaction costs in portfolio management," Annals of Operations Research, 2019.

[5] G. Araniti, M. Condoluci, A. Iera, L. Militano, and B. A. Pansera, "Analytical modeling of bargaining solutions for multicast cellular services," Electronic Journal of Differential Equations, vol. 2013, no. 165, pp. 1-10, 2013.

[6] L. S. Shapley, "A value for $n$-person games," in Contributions to the Theory of Games II, Annals of Mathematics Studies, H. W. Kuhn and A. W. Tucker, Eds., pp. 307-317, Princeton University Press, Princeton, NJ, USA, 1953.

[7] H. Moulin, "The separability axiom and equal-sharing methods," Journal of Economic Theory, vol. 36, no. 1, pp. 120-148, 1985.

[8] P. Sun, D. Hou, H. Sun, and T. Driessen, "Optimization implementation and characterization of the equal allocation of nonseparable costs value," Journal of Optimization Theory and Applications, vol. 173, no. 1, pp. 336-352, 2017.

[9] L. M. Ruiz, F. Valenciano, and J. M. Zarzuelo, "The least square prenucleolus and the least square nucleolus. Two values for TU games based on the excess vector," International Journal of Game Theory, vol. 25, no. 1, pp. 113-134, 1996.

[10] W. Wang, H. Sun, G. Xu, and D. Hou, "Procedural interpretation and associated consistency for the egalitarian Shapley values," Operations Research Letters, vol. 45, no. 2, pp. 164-169, 2017.

[11] L. S. Shapley, Additive and non-additive set functions, Ph.D. dissertation, Princeton University, Princeton, NJ, USA, 1953.

[12] E. Kalai and D. Samet, "On weighted Shapley values," International Journal of Game Theory, vol. 16, no. 3, pp. 205222, 1987.

[13] H. Yang, W. Wang, and Z. Ding, "The weighted surplus division value for cooperative games," Symmetry, vol. 11, no. 9, p. 1169, 2019.

[14] M. Malawski, “"Procedural” values for cooperative games," International Journal of Game Theory, vol. 42, no. 1, pp. 305-324, 2013.

[15] R. van den Brink and Y. Funaki, "Axiomatizations of a class of equal surplus sharing solutions for TU-games," Theory and Decision, vol. 67, no. 3, pp. 303-340, 2009.

[16] S. H. Tijs, "Bounds for the core and the $\tau$-value," in Game Theory and Mathematical Economic, pp. 123-132, NorthHolland Publishing Company, Amsterdam, The Netherlands, 1981.

[17] B. A. Pansera and M. Ferrara, " $n$-person cooperative games and geometrical aspects: a brief survey and new perspectives," 
Applied Mathematical Sciences, vol. 10, no. 17, pp. 833-843, 2016.

[18] M. Ferrara and B. A. Pansera, "A dynamic game for a sustainable supply chain management," in Complex Systems: Solutions and Challenges in Economics, Management and Engineering. Studies in Systems, Decision and Control, C. Berger-Vachon, A. Gil Lafuente, J. Kacprzyk, Y. Kondratenko, J. Merigó, and C. Morabito, Eds., vol. 125, pp. 331-348, Springer, Cham, Switzerland, 2018. 